\title{
La cara oscura del capital erótico. Capitalización del cuerpo y trastornos alimentarios. José Luis Moreno Pestaña. Madrid: Akal, 2016
}

\author{
Francisco Manuel Carballo Rodríguez \\ Universidad de Cádiz \\ franciscomanuel.carballo@uca.es
}

La publicación de La cara oscura del capital erótico. Capitalización del cuerpo y trastornos alimentarios completa un conjunto de trabajos, iniciados por su autor hace más de quince años sobre los trastornos alimentarios, la enfermedad mental y el cuerpo. Esta producción de ciclo largo, de referencia obligada para quienes se acerquen a la comprensión sociológica de dichas cuestiones, se ha sustanciado de diferentes maneras hasta Ilegar aquí. Se podrían diferenciar dos periodos en esa producción. Los primeros trabajos de José Luis Moreno Pestaña sobre el cuerpo, la alimentación y los trastornos alimentarios se completan en 2010 con la publicación de Moral corporal, trastornos alimentarios y clase social'. Los antecedentes de esa publicación se encuentran en una variedad de trabajos de carácter teórico y empírico, que vistos en su conjunto, conforman un sólido corpus de textos que nos orientan sobre el análisis original que, a propósito del cuerpo, su autor ha ido construyendo. La producción de ese primer periodo se nutre además de las investigaciones que José Luis Moreno Pestaña ha dedicado a la sociología de la enfermedad mental y de la desviación. Todo ello, como decía, está en el libro publicado por el CIS en 2010 y en la edición francesa ${ }^{2}$ del mismo que acaba de presentarse en la editorial Presses universitaires de Limoges. El volumen publicado en francés contiene lo fundamental de la versión original e introduce algunas novedades que actualizan los avances de las investigaciones más recientes. El texto lo cierra un interesante postfacio en el que el autor establece un diálogo con los responsables de la

1 Moreno Pestaña, J. L. (2010). Moral corporal, trastornos alimentarios y clase social. Madrid: CIS.

2 Moreno Pestaña, J. L. (2016). La classe du corps. Morale corporelle et troubles alimentaires. Limoges: Presses universitaires de Limoges. edición, los sociólogos Christophe Gaubert y MariePierre Pouly. En él, el autor reflexiona acerca de las cuestiones que son centrales en sus trabajos sobre el cuerpo, la enfermedad mental y los trastornos alimentarios, pero también sobre su producción en sociología de los intelectuales, en epistemología 0 sobre la conflictiva relación de las ciencias sociales con la filosofía y sus implicaciones científicas y políticas.

Algunos conceptos acuñados en toda esa serie de trabajos se han revelado como herramientas muy útiles para describir y analizar procesos sociales complejos. Pienso, por ejemplo, en la noción de "carrera corporal tensa", que abre un abanico de posibilidades de comprensión de la enfermedad mental, más allá de los estrechos límites que ofrece el espacio entre lo normal y lo patológico. En este sentido, otro concepto, el de "cronificación dulce", sirve para poner en relación el proceso por el que transitan las afectadas, en su relación con la enfermedad y con la oferta terapéutica disponible, en función de sus disposiciones, de sus condiciones sociales objetivas. Se trata, en suma, de una sociología de la enfermedad mental que observa las alteraciones de los hábitos compartidos, esto es, de las interacciones básicas que conforman el mundo social. Obviamente, esos procesos son vividos de formas muy desiguales en función de la posición social que se ocupe.

Un segundo periodo se cierra con el libro que aquí se presenta. El lector comprenderá, en lo que sigue, que hay una continuidad en el objeto de investigación. Una novedad destacable es la elección del terreno de estudio. Si, como decíamos antes, allí donde los hábitos se modifican de forma radical puede aparecer la enfermedad mental (los trastornos alimentarios), José Luis Moreno Pestaña trata de comprender qué sucede donde se imponen 
unas normas especialmente rígidas: el mercado de trabajo femenino. Novedad decía, porque la literatura académica sobre el cuerpo, especialmente en el caso de las mujeres, apenas ha prestado atención al mundo del trabajo. Parece que lo que sucediera, permítaseme el comentario al respecto, es que en la puerta de las empresas no solo se prohibiera la entrada de prácticas democráticas para las relaciones laborales, sino que también estuviese vetada la entrada al interés científico por lo que allí ocurre. No siempre es así, obviamente, pero en la jerarquía académica que opera en la elección de objetos y terrenos de investigación, al cuerpo - en especial al femenino - se le reservan espacios más nobles, más exclusivos, 0 bien más marginales, más minoritarios 0 más sórdidos, que aquellos en los que, parte de las mujeres de clase trabajadora desarrollan su actividad, como son las tiendas de moda, las perfumerías o los bares de copas. Todo ello está en este libro, aunque veremos que no exclusivamente, y creo que debe destacarse y reconocerse su doble valor. Valor, en primer lugar, desde un punto de vista de la elección científica, porque el autor nos proporciona una lección fundamental: analizando aquellas situaciones más conflictivas de la realidad social —las que pueden observarse empíricamente y no solo pensarse - es como pueden esclarecerse los mecanismos que reproducen y mantienen un orden social determinado. Esta enseñanza, que se desprende de los acercamientos del autor a sus objetos de investigación, está presente en la tradición sociológica y filosófica a la que pertenece, la de la escuela francesa, desde la epistemología de Bachelard hasta la sociología de Bourdieu o de Passeron ${ }^{3}$. Un segundo valor, esta vez de carácter político, se encuentra estrechamente unido a lo anterior. Se trata de hacer inteligibles los resultados de investigación de modo que pue-

3 Sobre esta cuestión es obligado dirigirse a la extensa introducción que José Luis Moreno Pestaña dedica a su traducción al castellano de la impresionante obra de Jean-Claude Passeron: Moreno Pestaña, J. L. (2010). "Estudio preliminar. Una filosofía de las ciencias históricas: presentación de la obra de Jean-Claude Passeron". En, Passeron, J.-C. El razonamiento sociológico. El espacio comparativo de las pruebas históricas (pp. 9-49). Madrid: Siglo XXI. dan ser utilizados para transformar estructuras que perduran a pesar de los efectos negativos que producen para un número importante de personas. Ambos valores podrían conjugarse evocando la conocida fórmula en la que Bourdieu dice de la sociología que "no merecería ni una hora de esfuerzo si tuviera que ser un saber de expertos reservado a expertos". La ciencia social que practica Moreno Pestaña es todo lo compleja que exigen los protocolos que a sí mismo se impone para no caer en la oscuridad de una práctica intelectual estéril que no sirva para la comprensión de la realidad. Práctica esta, por cierto, cada vez más frecuente en un buen número de trabajos académicos que se acorazan en dispositivos, bien estadísticos, bien teóricos, de escasa utilidad para la comprensión y la transformación social. Dicho queda y pasemos ahora al libro.

El libro está divido en siete capítulos, acompañados de una breve introducción, un apartado de conclusiones generales y un apéndice metodológico en el que el autor describe con detalle las condiciones institucionales y materiales de su investigación. En este último, encontramos un raro - por poco frecuente- ejercicio de reflexividad, de sociología de la sociología si se quiere, que informa al lector de las tensiones empíricas y teóricas que atravesaron a la investigación, que se empezó a gestar a finales del año 2011 y que se apoya en 45 entrevistas y en tres grupos de discusión. El material empírico aparece en el texto con mesura, con lo que proporciona un excelente ejemplo de administración razonada de la prueba, para ilustrar los análisis de los efectos de las condiciones laborales en la aparición de trastornos alimentarios. Todo ello está en los tres capítulos centrales del libro, del cuarto al sexto. En los tres primeros capítulos se propone una historia de la capitalización del cuerpo y en el séptimo y último, se ofrece un repertorio de posibilidades de cómo el cuerpo podría dejar de funcionar como un capital. Lo vemos a continuación.

En el primer capítulo se nos presenta una apasionante historia de la conversión del cuerpo en un capital. Dos momentos de dicha genealogía serán fundamentales para la argumentación posterior. En primer lugar, en el mundo griego clásico, José Luis 
Moreno Pestaña muestra cómo la belleza física no dependía de un único patrón y por lo tanto no podía convertirse en capital. ¿Las claves? El cuerpo no se encontraba entonces pedagógicamente disponible y en consecuencia no podía modificarse a voluntad. Además de lo anterior, en la Grecia clásica las formas de belleza eran plurales. Por último, la crítica de una parte del mundo intelectual al atletismo desconectaba la valoración ética del cuerpo de su identidad, de su valor moral. Para Sócrates o Eurípides, la inversión de tiempo y esfuerzo en hazañas corporales suponía renunciar al cultivo de competencias culturales y mermaba las capacidades políticas de los atletas. En suma, la morfología corporal, el vestido y la identidad no reflejaban las diferencias sociales. Esto no quiere decir que en la sociedad griega del momento dichas diferencias no fuesen importantes, que lo eran, quiere decir que no se encarnaban. El segundo momento que nos interesa destacar acontece entre la mitad del siglo xIx y principios del siglo xx, pues será entonces cuando se empiecen a estabilizar determinados modelos corporales estandarizados. El proceso se culmina gracias a la extensión de los regímenes de adelgazamiento durante el siglo xvII y el recurso creciente a la cosmética durante el siglo xVIII. Ahora sí, con la belleza unificada en un único patrón y con el concurso de la legitimación sanitaria de la delgadez, la encarnación de las diferencias sociales será un hecho. De este modo, los prototipos corporales, los recursos eróticos, comienzan a identificarse con una especie de capital, susceptible de ser acumulado por los sujetos, que se sirven de él. Pero para que eso sea posible, para que un recurso se convierta en un capital es necesario que se inserte en un mercado. De eso se ocupa el segundo capítulo.

Me centraré aquí en la que es la justificación de la tesis que se defiende en el libro: el capital erótico es una especie de capital cultural. Las competencias estéticas vinculadas a la belleza, nos explica el autor, pueden considerarse una especie de capital cultural en alguna de sus tres formas, siguiendo el esquema clásico de Bourdieu: en su forma incorporada (el sujeto interesado debe querer adquirirlo y hacer lo posible para ello), en su forma objetivada (puede recibirse, como una herencia, pero es fundamental que quien lo recibe tenga las competencias para valerse de él) y, por último, puede darse en su forma institucionalizada, que es aquella que proporcionan los títulos educativos. En su formulación el autor se distancia de Catherine Hakim, quien pretende que la noción de capital erótico se sume a la tríada propuesta por Bourdieu de capital económico, capital cultural y capital social, como una especie de capital diferente. Llegados a este punto, cabría preguntarse por cuáles son aquellos mercados, aquellos espacios, en los que los recursos estéticos pueden convertirse en capital erótico. Para responder a esta cuestión, el autor, con la ayuda de Randalls Collins y de Viviana Zelizer, explica que los capitales necesitan de espacios específicos donde poder activarse. Un paso más en la argumentación nos adentrará en la relación de los trastornos alimentarios con el capital erótico. El capítulo tercero traza las líneas que delimitan las fuerzas estructurales que hacen posible la presencia de enfermedades autónomas relacionadas con la alimentación y con la apariencia física. En los espacios en los que el capital erótico se activa, existen nichos ecológicos —el autor se apoya aquí en los modelos de análisis de la enfermedad mental de lan Hacking - en los que, a través de las interacciones, la enfermedad aparece, se desarrolla y permanece. En los tres capítulos que siguen, la teoría desarrollada hasta aquí será puesta a prueba en un terreno al que ya se hizo alusión, el mercado laboral femenino.

La primera de las aportaciones, al respecto de los vínculos entre presión estética —aumento del valor del capital erótico en los ambientes laborales muy feminizados - y el riesgo de aparición de conductas desviantes asociadas a la conducta alimentaria, es la constatación de la preocupación, cada vez más temprana, por el peso y la apariencia sexual. Esta tendencia se extendió a partir de los años 80 y supuso un retroceso en los avances de la crítica feminista de las décadas de los 60 y 70. La liberación sexual se había sustituido por una progresiva sexualización de la mujer. Este fenómeno, conocido como el backclash, o la "torcedura de bastón", se extendería y colonizaría la experiencia de una generación de mujeres. En los casos descritos y analizados en este libro, se comprueba cómo, a partir de la década de los 90, las exigencias estéticas 
en el puesto de trabajo comenzaban a ser cada vez más altas, no sin conflictos entre las distintas generaciones que convivían en esos espacios. A la extensión y la valorización del capital erótico entre las trabajadoras de ciertos sectores, le sigue el efecto que la presencia masiva de dicho capital juega en las interacciones cotidianas. Los juegos estéticos están muy presentes en dichas interacciones y sirven para cultivar el capital erótico. Con el recurso de dicha noción de juegos estéticos, el autor consigue aislar un repertorio de modos de relacionarse en los ambientes laborales —entre las propias trabajadoras y también entre las trabajadoras y las clientas-. Este conjunto de juegos permite observar, no solo formas de sometimiento de las trabajadoras a las normas impuestas por la empresa, sino también formas de cultivo de una forma específica de capital cultural como el capital erótico mediante las que se manifiesta una expresión de competencia intelectual. Un último factor a considerar es el de la relación entre la presión corporal y el mercado de trabajo más cualificado. En este sentido, el autor concluye, que de una manera general podría constatarse que la presión erótica específica disminuye a medida que el capital cultural aumenta.

Para terminar, en el capítulo siete se enfrenta la difícil tarea de pensar en las posibles formas de salida de las prácticas más perjudiciales asociadas a la acumulación y el mantenimiento del capital erótico. De qué modo podría, en definitiva, politizarse la experiencia del sufrimiento corporal. Tres formas de contestación podrían hacer frente a la "cara oscura" del cultivo del capital erótico. Las tres tareas requieren un esfuerzo colectivo importante. Se trataría de contestar a los procesos que fundaban la encarnación de las diferencias sociales. Para ello, habría que cuestionar los modelos únicos de belleza, comprender que el cuerpo no se encuentra disponible y asumir, en fin, que el cuerpo no nos dice nada sobre el valor moral de un individuo.

Sobre las aportaciones de este libro a las diferentes ramas del conocimiento sociológico en las que se adentra, tendrán que pronunciarse los especialistas. El libro no elude ningún debate e interpela autores y teorías muy variadas. De su existencia o no y del vigor de esos debates podrán extraerse conclusiones del momento que atraviesan las ciencias sociales en nuestro país. Por otro lado, para el lector interesado en la literatura sociológica, el libro ofrece una cantidad extraordinaria de información con la que dispondrá de una historia clara sobre las transformaciones del cuerpo y las tensiones científico-técnicas y políticas que la recorren. Me atrevo a decir, por último, que este libro puede ser leído y utilizado con intereses muy distintos, casi como un manual de teoría y de metodología de las ciencias sociales. También, y eso obvia decirlo a estas alturas, como fuente de herramientas teóricas para pensar la transformación social. 